\title{
Intersection of autophagy with pathways of antigen presentation
}

\author{
Natalie L. Patterson, Justine D. Mintern $\bowtie$ \\ Department of Biochemistry and Molecular Biology, The University of Melbourne, Parkville, Victoria 3010, Australia \\ $\triangle$ Correspondence: jmintern@unimelb.edu.au \\ Received September 4, 2012 Accepted October 2, 2012
}

\begin{abstract}
Traditionally, macroautophagy (autophagy) is viewed as a pathway of cell survival. Autophagy ensures the elimination of damaged or unwanted cytosolic components and provides a source of cellular nutrients during periods of stress. Interestingly, autophagy can also directly intersect with, and impact, other major pathways of cellular function. Here, we will review the contribution of autophagy to pathways of antigen presentation. The autophagy machinery acts to modulate both $\mathrm{MHCl}$ and $\mathrm{MHCll}$ antigen presentation. As such autophagy is an important participant in pathways that elicit host cell immunity and the elimination of infectious pathogens.
\end{abstract}

KEYWORDS autophagy, antigen presentation, antigen presenting cells, dendritic cells, $\mathrm{MHCl}, \mathrm{MHCll}$.

\section{AUTOPHAGY}

Macroautophagy, hereafter referred to as autophagy, describes a process of cellular self-digestion conserved in eukaryotic cells (Yang and Klionsky, 2010). Autophagosomes, double-membrane bound vesicles, envelope components of the cytoplasm (Yang and Klionsky, 2010), such as misfolded proteins and damaged organelles. Autophagosomes fuse with lysosomes, resulting in the degradation of autophagosomal contents. Autophagy serves several important cellular functions. It removes unwanted proteins from the cytosol, preventing their accumulation and also provides cells with additional nutrients upon release of amino acids after proteolysis. In addition, autophagy establishes a trafficking pathway from the cytosol to the endolysosomal compartments. Thus autophagy has been shown to be crucial to the maintenance and survival of cells. It is therefore not surpris- ing that autophagy has been associated with several important biological processes and diseases (Levine and Kroemer, 2008). Autophagy plays a major role in generating robust immunity (Mintern and Villadangos, 2012) with participation in the clearance of pathogens, survival of immune cells and importantly in pathways of antigen presentation, the focus of this review.

To date, approximately thirty autophagy related genes (Atg) have been discovered through Saccharomyces cerevisiae yeast studies that have aided the identification of their mammalian orthologs (Klionsky et al., 2003; Suzuki and Ohsumi, 2007). During normal cellular conditions, autophagy occurs at a basal level and is regulated by a network of stress sensors, sensitive to alterations in the cellular environment. The mammalian target of rapamycin complex 1 (mTORC1), is the major regulator of autophagy, and when active, suppresses autophagosome formation. Cues such as nutrient deprivation or damaged organelles allow upstream stress sensors to phosphorylate components of mTORC1, inhibiting its regulatory actions and upregulating autophagy (Jung et al., 2010). In brief, the autophagy pathway involves three major steps: initiation of the autophagosome, elongation of the double membrane, maturation and fusion of the autophagosome with the lysosome to form an autolysosome. Initiating autophagy, mTORC1 inhibition causes dephosphorylation and activation of unc-51 like kinase (ULK) 1 and 2. An ULK complex is formed that interacts with the isolation membrane, the enveloping membrane that develops from the pre-autophagosomal structure (PAS) (Hara et al., 2008; Jung et al., 2009). Atg9, a transmembrane protein, is also a critical protein recruited to the site of autophagosome synthesis (Orsi et al., 2012). In addition to mTORC1, autophagy is also regulated by vacuolar protein sorting 34 (Vps34), the class III phosphatidylinositol 3-kinase (PI3K) that localizes to the isolation membrane where it synthesizes phosphatidylinositol 3-phosphatate (PI3P) (Burman and Ktistakis, 2010). The 
origin of the PAS is currently a point of contention. While de novo membrane synthesis in the cytosol has been hypothesized to initiate autophagosome formation, several studies suggest it can originate from other sites including the endoplasmic reticulum (ER), mitochondria and plasma membrane (Weidberg et al., 2011). Ubiquitin ligase-like systems permit the elongation and formation of an autophagosome. Atg12 is conjugated to Atg5 by Atg7 (an E1-like ligase) and Atg10 (an E2-like ligase) (Mizushima et al., 1998; Shintani et al., 1999). Atg16L1 is then conjugated to Atg5 and together this complex mediates the elongation of the autophagosomal membrane (Ohsumi, 2001). In addition, microtubule-associated protein light chain 3 (LC3) is cleaved by cysteine protease Atg4 to become LC3-I. Subsequently, Atg7 and Atg3, also an E2-like ligase, conjugate free cytosolic LC3-I to phosphatidylethanolamine (PE) to become LC3-II-PE that associates with autophagosomes (Tanida et al., 2004). Finally, autophagosomes travel to the endolysosomal compartment, whereupon fusion with lysosomes, the autophagosomal contents are degraded.

\section{MONITORING AUTOPHAGY}

Autophagy is a dynamic process that is not simple to measure. As such, there are several methods and tools that have been described to measure autophagy. Identification of autophagosomes within cells is a useful strategy to determine whether cells are undergoing autophagy. Autophagosomes are double-membraned structures that can be visualised by electron microscopy (Baba et al., 1994) (Klionsky and Ohsumi, 1999). A widely utilised marker of autophagy is microtubule-associated protein light chain 3 (LC3) (Tanida et al., 2004). As mentioned, this protein exists in two isoforms, LC3-I and LC3-II. Notably, LC3-II associates with both the internal and external autophagosomal membranes and upon autophagosome-lysosome fusion, LC3-II is degraded (Kabeya et al., 2000). Consequently, LC3-II is a useful marker for measuring autophagy-mediated degradation. Upon treatment of cells with pharmacological inhibitors of lysosomal proteolysis (which prevents LC3-II degradation following autolysosome formation), LC3-II accumulation can be detected. Comparison of LC3-II accumulation between cells that can and cannot undertake lysosomal proteolysis permits a relative quantification of autophagy-mediated degradation.

To determine the impact of autophagy on cellular processes, prevention of the autophagy pathway, via either inhibition or knockdown, is required. Wortmannin and 3-methyladenine (3-MA) inhibit PI3Ks (Blommaart et al., 1997) (Seglen and Gordon, 1982) that are essential for autophagosome formation. However, PI3Ks are not exclusive to the autophagy pathway and so conclusions about the role of autophagy upon PI3K inhibition should be treated with caution. A more comprehensive approach to studying autophagy is by the knock down of an Atg family member. Many studies use siRNA and shRNA knockdowns of Atg genes. Atg deficient mice are available either as knockout or loxp flanked genes including Atg3 (Sou et al., 2008; Jia and He, 2011), Atg9a (Saitoh et al., 2009), Atg16L1 (Saitoh et al., 2008), Atg5 (Kuma et al., 2004) and Atg7 (Komatsu et al., 2005). In addition, Vps 34 conditional knockout mice have been generated (McLeod et al., 2011) (Willinger and Flavell, 2012). Finally, green fluorescent protein (GFP)-LC3 reporter mice can be used to monitor autophagy in vivo (Mizushima et al., 2004). In summary, the complexity of the autophagy pathway requires that several of the outlined methods be utilised to carefully evaluate autophagy in any given cell type.

\section{ANTIGEN PRESENTATION}

To combat infectious pathogens, $T$ cells recognise antigen presented in the context of major histocompatibility complex $(\mathrm{MHC})$ molecules. Antigen presentation refers to the pathways by which antigen is loaded into MHC molecules. Antigen presentation is critical to initiating an immune response that will ensure robust pathogen clearance from the infected host. Cells express $\mathrm{MHC}$ class I (MHCl) and $\mathrm{MHC}$ class II (MHCII) molecules. $\mathrm{MHCl}$ molecules are expressed by all cell types and are recognised by $\mathrm{CD} 8^{+} \mathrm{T}$ lymphocytes. In contrast, $\mathrm{MHCll}$ molecules are limited in their expression to B cells, macrophages and dendritic cells, cell types that are collectively known as antigen presenting cells (APC). MHCll loaded with antigen is recognised by $\mathrm{CD}^{+} \mathrm{T}$ cells. There are three major pathways of antigen presentation. The classical pathway of $\mathrm{MHCl}$ presentation involves presenting intracellular antigen that is synthesised by the cell itself. In this case, antigen is degraded into peptides by the proteasome, with further trimming provided by various peptidases. Peptides are transported into the endoplasmic reticulum and loaded into newly synthesised $\mathrm{MHCl}$ molecules for surface display. All cell types can present antigen via the classical $\mathrm{MHCl}$ antigen presentation pathway. Interestingly, exogenous antigen acquired from outside the cell can also be presented by $\mathrm{MHCl}$. This pathway is known as "cross-presentation" and can only be performed by specialised APC. Finally, MHCIl antigen presentation involves the presentation of exogenous antigen that is captured by APC. In this case antigen loading occurs in the endolysosomal compartments where antigens are degraded by lysosomal proteases and loaded into $\mathrm{MHCll}$ molecules that have been trafficked there from the ER. Here, we will review the contribution of autophagy to classical $\mathrm{MHCl}, \mathrm{MHCll}$ and $\mathrm{MHCl}$ cross-presentation pathways (Fig. 1).

\section{AUTOPHAGY IN MHC CLASS II ANTIGEN PRESENTATION}

$\mathrm{CD}^{+} \mathrm{T}$ cells recognise antigen presented by MHCll mole- 


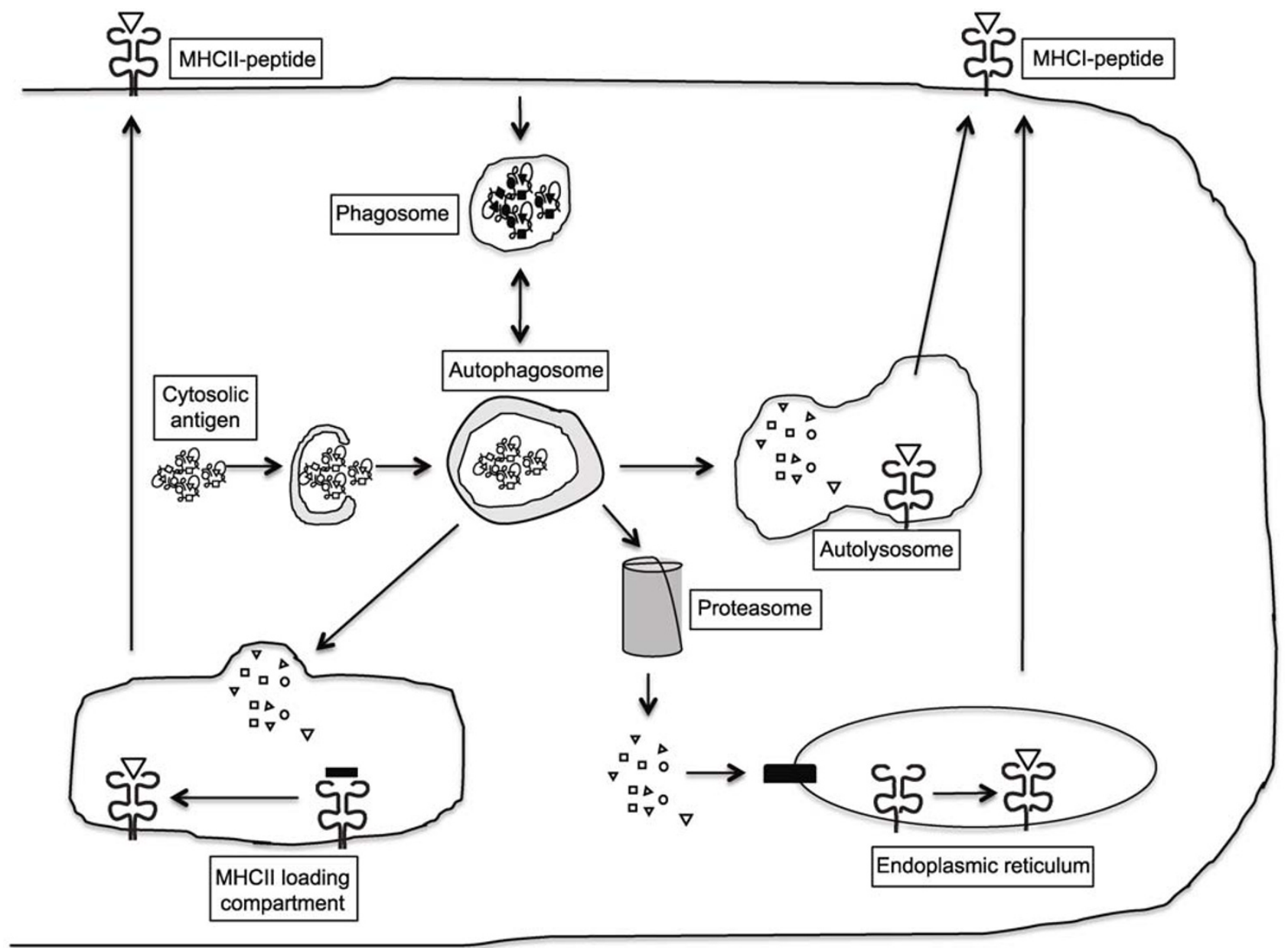

Antigen presenting cell

Figure 1. Intersection of autophagy with $\mathrm{MHCl}$ and $\mathrm{MHCll}$ antigen presentation pathways. For $\mathrm{MHCll}$ antigen presentation, autophagy traffics cytosolic antigen to MIIC loading compartments. For $\mathrm{MHCl}$ antigen presentation, antigen may escape the autophagosome for access to the classical $\mathrm{MHCl}$ presentation pathway or $\mathrm{MHCl}$ molecules may be loaded with antigen in an autolysosome compartment. For $\mathrm{MHCl}$ cross-presentation, autophagosomes or the autophagy machinery may intersect with phagosomes containing exogenous antigen.

cules. Interestingly, numerous examples exist of self and foreign intracellular antigens that are presented by $\mathrm{MHCll}$ molecules (Nimmerjahn et al., 2003; Dorfel et al., 2005; Paludan et al., 2005; Schmid et al., 2007; Riedel et al., 2008; Jagannath et al., 2009). How do these antigens access endosomal MHCIl loading compartments (termed MIIC)? Given the trafficking route of autophagosomes from cytosol to lysosomes, autophagy is implicated in the delivery of cytosolic antigen to the MIIC. Indeed, immature and mature human monocyte-derived dendritic cells (DC) undergo constitutive autophagy with the majority of autophagosomes fusing with MIIC (Schmid et al., 2007). For several antigens, a direct role for autophagy in $\mathrm{MHCll}$ antigen presentation has been demonstrated. MHCll presentation of cytosolic neomycin phosphotransferase (NeoR) antigen in Epstein Barr virus (EBV) transformed $B$ cell lines is greatly reduced in the presence of autophagy inhibitors 3-MA and wortmannin (Nimmerjahn et al., 2003). In addition, siRNA knockdown of Atg12 impairs NeoR specific $\mathrm{CD}^{+} \mathrm{T}$ cell proliferation in response to nuclear-located NeoR (Riedel et al., 2008). Inhibition of autophagy via 3-MA treatment or siRNA knockdown of Atg12 also reduces $\mathrm{MHCll}$ antigen presentation of Epstein-Barr nuclear antigen 1 (EBNA-1) to $\mathrm{CD}^{+} \mathrm{T}$ cells (Paludan et al., 2005). Another example of a cytosolic antigen presented by $\mathrm{MHCl}$ is the tumour antigen mucin 1. When mucin 1 is introduced into the cytosol of human monocyte-derived DC, mucin 1specific $\mathrm{CD}^{+} \mathrm{T}$ cell proliferation is induced (Dorfel et al., 2005). Mucin 1-specific $\mathrm{CD}^{+}{ }^{+} \mathrm{T}$ cells do not proliferate as efficiently following 3-MA or wortmannin treatment of mucin 1-expressing DC, suggesting a requirement for autophagosomes in the presentation of cytosolic mucin 1 by MHCIl molecules (Dorfel et al., 2005). Finally, starvation-induced autophagy shifts $\mathrm{MHCll}$ ligand selection to increase the display of resident intracellular peptides (Dengjel 
et al., 2005). Therefore, autophagy contributes to the presentation of antigen by $\mathrm{MHCll}$ and consequently impacts $\mathrm{CD} 4^{+}$ $T$ cell immunity.

Interestingly, there is evidence to suggest that enhancement of autophagy, or the targeting of antigen to autophagosomes, can enhance MHCII antigen presentation. Fusing influenza matrix protein 1 (MP1) to LC3 targets MP1 to autophagosomes and improves MP1 presentation to, and activation of, antigen-specific $\mathrm{CD}^{+}{ }^{+} \mathrm{T}$ cells (Schmid et al., 2007). Furthermore, upregulation of autophagy with rapamycin in murine macrophages and $C D 11 \mathrm{c}^{+}$conventional DC (cDC) infected with Mycobacterium tuberculosis (MTb) increases $\mathrm{MHCll}$ antigen presentation of the MTb secreted antigen, Ag85B (Jagannath et al., 2009). Vaccination of mice with rapamycin-treated, MTb-infected $\mathrm{CDC}$ enhances primary antigen-specific $\mathrm{CD} 4^{+} \mathrm{T}$ cell responses and upon challenge with MTb, recipients from the rapamycin-treated group mount superior $\mathrm{CD}^{+} \mathrm{T}$ cell responses and have lower bacterial loads compared to the untreated controls (Jagannath et al., 2009). Together, these results demonstrate that access to, and enhancement of, the autophagy pathway upon infection can improve immune responses. As such, the autophagy pathway represents a new and exciting route for vaccination.

In addition to its role in endogenous MHCll antigen presentation, the autophagy machinery is also implicated in exogenous $\mathrm{MHCll}$ antigen presentation. $\mathrm{CD} 11 \mathrm{c}^{+} \mathrm{CDC}$ from Atg5-deficient foetal liver reconstituted mice infected with either ovalbumin-herpes simplex virus-2 (OVA-HSV-2) or OVA-Listeria monocytogenes, are unable to efficiently prime adoptively transferred OVA-specific $\mathrm{CD}^{+}{ }^{+} \mathrm{OT}$-II T cells and induce OT-II T cell proliferation in vivo (Lee et al., 2010). Furthermore, protective immune responses require Atg5 expression by CDC. The authors rule out a role for Atg5 in surface expression of MHCII, CD40 and CD86, the production of inflammatory cytokines or DC migration (Lee et al., 2010). The absence of Atg5 does not affect lysosome function, instead Atg5-deficient CDC are unable to present antigen on $\mathrm{MHCll}$ molecules due to the reduced ability of phagosomes to fuse with lysosomes (Lee et al., 2010). Of note, double-membrane bound autophagosomes are not observed during this process and the authors hypothesise involvement of Atg5 in a non-canonical autophagy pathway. Together, this suggests that the Atg protein Atg5, but not canonical autophagy, is required for exogenous antigen processing and presentation by MHCII molecules (Lee et al., 2010).

In contrast, there are examples where autophagy does not contribute to $\mathrm{MHCll}$ antigen presentation of intracellular antigen. Comber et. al. have demonstrated that while autophagy is upregulated in influenza A virus infected cells, autophagy does not enhance $\mathrm{MHCll}$ antigen presentation of the site 1 (S1) epitope of hemagglutinin (HA) (Comber et al., 2011). The targeting of S1 to autophagosomes, either via S1 conjugation to LC3 or to NeoR, a model antigen, shown previously to be presented by MHClI molecules (Nimmerjahn et al.,
2003), does not enhance S1-specific $\mathrm{CD}^{+} \mathrm{T}$ cell responses (Comber et al., 2011). In addition, shRNA knockdown of Atg7 in bone marrow-derived DC does not affect CD4+ $\mathrm{T}$ cell interferon-y (IFNy) secretion in response to S1 (Comber et al., 2011). Chaperone-mediated autophagy (CMA) is a potential alternative route that may deliver antigen to the $\mathrm{MHCll}$ antigen presentation pathway. CMA describes the selective delivery of cytoplasmic peptides and proteins directly into the lumen of the lysosome (Orenstein and Cuervo, 2010; Li et al., 2011b). The CMA machinery, including heat shock cognate protein of $70 \mathrm{kDa}$ (hsc70) that selects peptide substrates, and multimeric lysosomal-associated membrane protein $2 \mathrm{~A}$ (LAMP-2A) complex, facilitates the entry of peptide into the lysosome (Orenstein and Cuervo, 2010; Li et al., 2011b). $\mathrm{MHCll}$ antigen presentation of cytosolic glutamate decarboxylase in human $\mathrm{B}$ lymphoblastoid cells is reduced following knockdown of LAMP-2 or hsc70 (Zhou et al., 2005). In addition, overexpression of $L A M P-2 A$ or $h s c 70$ increases $\mathrm{MHCll}$ presentation of cytosolic glutamate decarboxylate. Therefore, while autophagy is a viable route for delivering intracellular antigen to $\mathrm{MHCll}$ loading compartments, it is not the only pathway by which to do so.

\section{AUTOPHAGY IN CLASSICAL MHC CLASS I ANTIGEN PRESENTATION}

$\mathrm{CD}^{+} \mathrm{T}$ cells recognise antigen presented by $\mathrm{MHCl}$ molecules. Autophagy participation in classical $\mathrm{MHCl}$ antigen presentation is not obvious, given that the endogenous $\mathrm{MHCl}$ antigen presentation pathway does not intersect with the autophagosomal route. As described, for classical $\mathrm{MHCl}$ antigen presentation, antigen is degraded in the cytosol by the proteasome and transported into the ER where it is loaded on newly synthesized $\mathrm{MHCl}$ molecules. Therefore, for most antigens, autophagy plays little to no role in their presentation by MHC class I. For example, siRNA knockdown of Atg12 in an EBV-transformed bone marrow-derived lymphoblastoid cell line does not impair antigen-specific $\mathrm{CD} 8^{+} \mathrm{T}$ cell responses (Paludan et al., 2005). Autophagy inhibition with 3-MA or wortmannin has no effect on tyrosinase antigen presentation by HLA (Nimmerjahn et al., 2003), and targeting the influenza matrix protein 1 (MP1) to autophagosomes does not enhance IFNy secretion by antigen-specific $\mathrm{CD} 8^{+} \mathrm{T}$ cells (Schmid et al., 2007).

Despite the large body of evidence that suggests autophagy does not play a role in $\mathrm{MHCl}$ antigen presentation, there are some reports to the contrary. The autophagy pathway can impact $\mathrm{MHCl}$ antigen presentation under specific circumstances. A pertinent example is the role of autophagy in facilitating $\mathrm{MHCl}$ presentation by herpes simplex virus 1 (HSV-1) infected macrophages, 8-12 h after infection (English et al., 2009). At these time points, treatment of HSV-1 infected macrophages with bafilomycin A (an inhibitor of lysosomal acidification), 3-MA or knockdown of Atg5, im- 
pairs HSV-1 glycoprotein B (gB)-specific $\mathrm{CD}^{+} \mathrm{T}$ cell activation, indicating that at this stage of infection both autophagosomes and lysosomal degradation are required for $\mathrm{gB}$ $\mathrm{MHCl}$ antigen presentation (English et al., 2009). Using fluorescence and electron microscopy, LC3+ autophagosomes are observed to originate from the nuclear envelope containing HSV-1 particles (English et al., 2009). The authors suggest that $\mathrm{gB}$ processing begins in the autophagosome after which gB peptides escape to the cytosol for further processing by the proteasome and entry into the $\mathrm{MHCl}$ antigen presentation pathway. Another example of a viral protein that requires autophagy for $\mathrm{MHCl}$ presentation is human cytomegalovirus (HCMV) latency associated protein pUL138 (Tey and Khanna, 2012). In this case, $\mathrm{MHCl}$ presentation is independent of the transporter associated with antigen processing (TAP). Inhibition of the proteasome with lactacystin or epoxomicin, or inhibition of ER aminopeptidases, does not affect pUL138-specific $\mathrm{CD}^{+} \mathrm{T}$ cell responses. In contrast, chloroquine treatment, utilised to reduce lysosomal protease activity, does impair pUL138-specific $\mathrm{CD} 8^{+} \mathrm{T}$ cell responses. Knockdown of autophagy with either 3-MA or Atg12 siRNA reduces pUL138-specific $\mathrm{CD} 8^{+} \mathrm{T}$ cell responses, while heat shock induced upregulation of autophagy enhances these responses (Tey and Khanna, 2012). Interestingly, unlike HSV-1 gB, Tey et al. hypothesize that pUL138 degradation and subsequent generation and loading of the peptide into $\mathrm{MHCl}$ occurs in the autophagolysosome, where antigen does not access to the cytosol. A similar pathway of $\mathrm{MHCl}$ antigen presentation has also been described for an epitope derived from respiratory syncytial virus, where $\mathrm{MHCl}$ antigen presentation was abrogated in the presence of 3-MA treatment (Johnstone et al., 2012). Therefore autophagy-mediated $\mathrm{MHCl}$ presentation is a relevant pathway of antigen presentation for several virus-associated antigens and may, or may not, involve the classical $\mathrm{MHCl}$ antigen presentation route. Whether autophagy also participates in $\mathrm{MHCl}$ presentation of endogenous antigens or epitopes derived from non-viral sources remains to be determined.

Finally, autophagy may also participate in $\mathrm{MHCl}$ surface turnover. Pharmacological inhibition of autophagy with 3-MA, bafilomycin A, chloroquine or wortmannin, or siRNA knockdown of beclin 1, Atg5 or Atg7 in murine macrophages, increases surface expression of $\mathrm{MHCl}$ molecules ( $\mathrm{Li}$ et al., 2010). Rapamycin treatment of B16 tumour cells promotes the localisation of $\mathrm{MHCl}$ molecules with autophagosomes and reduces $\mathrm{MHCl}$ cell surface expression (Li et al., 2010). In contrast, IFNy, in combination with rapamycin, prevents $\mathrm{MHCl}$ co-localization with autophagosomes and enhances surface expression of $\mathrm{MHCl}$ molecules. IFNy and rapamycin treatment of B16 tumour cells enhances antigen-specific cytotoxic $T$ lymphocyte (CTL) killing of tumour cells, with the siRNA knockdown of beclin 1 in B16 tumour cells significantly reducing this effect (Li et al., 2010). Therefore, it is postulated that surface $\mathrm{MHCl}$ levels can be regulated via auto- phagy-mediated degradation.

\section{MHCI CROSS-PRESENTATION AND AUTOPHAGY}

Cross-presentation describes the presentation of exogenous antigen by $\mathrm{MHCl}$ molecules (Bevan, 1976). It is essential for inducing CTL responses against pathogens that do not directly infect professional APC. During cross-presentation, exogenous antigen is phagocytosed by $D C$ and enters the $\mathrm{MHCl}$ antigen processing pathway. The exact mechanism or mechanisms of how this occurs is unknown. Proposed models of cross-presentation include the delivery of exogenous antigen from the phagosome into the cytosol and the subsequent entry of antigen into the classical $\mathrm{MHCl}$ processing pathway, or recycling $\mathrm{MHCl}$ molecules from the cell surface to endosomes/late endosomes for peptide loading (Segura and Villadangos, 2011; Joffre et al., 2012).

A role for autophagy in efficient cross-presentation is controversial. Experiments by Lee et. al. suggest that autophagy does not affect $\mathrm{MHCl}$ cross-presentation in murine DC (Lee et al., 2010). To investigate cross-presentation, the ability of wildtype DC versus Atg5-deficient DC to cross-present either soluble OVA, or lethally irradiated OVA-pulsed MHCl-deficient splenocytes, to CD8 ${ }^{+}$OVA-specific OT-I T cells was examined. Atg5-deficient DC display no apparent defects in cross-presentation of either soluble or cell-associated OVA (Lee et al., 2010). In contrast to this finding, autophagy participates in the cross-presentation of nanoparticle-associated OVA. $\alpha \mathrm{Al}_{2} \mathrm{O}_{3}$ nanoparticles act as adjuvants with their con-

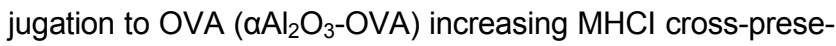
ntation of OVA by bone marrow-derived DC (Li et al., 2011a). This is evidenced by enhanced OT-I T cell responses in vitro and in vivo compared to DC cultured with OVA alone. Delivery of $\mathrm{aAl}_{2} \mathrm{O}_{3}$-coated autophagosomes to tumour-bearing mice improves OT-I T cell responses, and unlike the administration of OVA with Alum (a widely used adjuvant), abrogates tumour growth (Li et al., 2011a). Notably, $\alpha \mathrm{Al}_{2} \mathrm{O}_{3}-\mathrm{OVA}$ nanoparticles co-localise with LC3+ autophagosomes in DC, while OVA alone does not. Inhibiting autophagy with 3-MA, wortmannin or siRNA knockdown of beclin 1, prevents cross-presentation of OVA when conjugated to $\mathrm{\alpha Al}_{2} \mathrm{O}_{3}$. The authors propose a direct role for the autophagosome itself as a compartment for cross-presentation (Li et al., 2011a). The notion that autophagy facilitates cross-presentation is supported by the observation that the autophagy machinery promotes the transit of Aspergillus conidia associated antigen from the early endosome to a Rab14+ cross-presenting compartment (De Luca et al., 2012). Therefore, the role of autophagy in cross-presentation remains to be further explored in detail.

Autophagosomes can serve as immunogenic compartments that have efficient access to the cross-presentation pathway. Dying cells from bax/bak-deficient mouse embryonic fibroblasts (MEFs) display enhanced autophagy. Infec- 
tion of these cells with influenza A virus facilitates enhanced cross-presentation of influenza A virus-derived antigen to $\mathrm{CD}^{+} \mathrm{T}$ cells (Uhl et al., 2009). siRNA knockdown of Atg5 in bax/bak-deficient MEFs abrogates this response (Uhl et al., 2009). In addition, rapamycin treatment of the human melanoma cell line FEMX enhances cross presentation of tumour antigen gp100, while this is abrogated by preventing autophagy with 3-MA or wortmannin, beclin 1 shRNA and Atg12 siRNA knockdown (Li et al., 2008). LC3+ autophagosomes purified from OVA-expressing fibroblasts also elicit crosspresentation when cultured with DC (Li et al., 2008). Collectively, these observations suggest that autophagosomes serve as effective vehicles for the delivery of exogenous antigen for the cross-presentation pathway. These observations may stem from the finding that autophagy is required for the engulfment of dying cells (Qu et al., 2007). Atg5 and beclin 1-deficient embryos are not phagocytosed as efficiently as autophagy competent cells. Autophagy deficient cells do not readily express cell surface membrane-bound phosphatidylserine, and secreted lysophosphotidylcholine, which entice and attract APC for phagocytosis (Qu et al., 2007). Therefore, autophagy may enhance cross-presentation by promoting efficient phagocytosis of exogenous antigen.

\section{AUTOPHAGY IN THE THYMUS}

In the thymus, antigen presentation pathways play a critical role in the selection of $\mathrm{CD}^{+}$and $\mathrm{CD}^{+}{ }^{+}$lymphocytes. Cortical (cTEC) and medullary (mTEC) thymic epithelial cells present $\mathrm{MHCll}$ restricted self antigen for the positive and negative selection of $\mathrm{CD}^{+} \mathrm{T}$ cells, respectively. These cells have poor phagocytic capabilities, and therefore it was hypothesised that self antigen presented to CD4 single positive thymocytes was largely of endogenous origin. How antigen accessed the MHCII antigen presentation pathway in TEC was until recently, largely unknown. Notably, CTEC and mature mTEC constitutively undergo high levels of autophagy (Nedjic et al., 2008; Kasai et al., 2009; Sukseree et al., 2012). In addition, LC3-II co-localizes with MHCII, MHCII with class II-associated invariant chain peptide, H2-DM and LAMP-1 in IFNy treated cTEC and mTEC (Kasai et al., 2009), suggesting that autophagosomes fuse with MIIC. Quantitative analysis of peptides presented in MHCII molecules by Atg5-deficient CTEC reveals a skewed distribution of peptide-loaded MHC ligands compared to wildtype controls (Nedjic et al., 2008). This supports a role for autophagy in the delivery of some, but not all, self peptides for MHCII antigen presentation in cTEC. Furthermore, $\mathrm{CD} 4^{+} \mathrm{T}$ cell tolerance induction by Atg5-deficient mTEC is impaired, with autoimmunity elicited in peripheral tissues (Nedjic et al., 2008). Collectively, these findings demonstrate the in vivo requirement of autophagy in the positive and negative selection of $\mathrm{CD}^{+} \mathrm{T}$ cells. In contrast, Sukseree et. al. demonstrate that knockdown of Atg7 in TEC does not promote autoimmunity in peripheral organs
(Sukseree et al., 2012). Whether this contradicts the role of autophagy in $\mathrm{CD}^{+} \mathrm{T}$ cell selection in the thymus or simply endorses the finding that some but not all peripheral antigen access the autophagy pathway for MHCll antigen presentation remains to be determined. While autophagy is primarily thought to participate in $\mathrm{CD}^{+} \mathrm{T}$ cell selection, its role, if any, in $\mathrm{CD}^{+}{ }^{+}$cell selection remains to be defined.

\section{REGULATION OF AUTOPHAGY}

Autophagy is regulated in APC by various stimuli that are provided during immune responses. These stimuli include cytokines and ligands that are recognised by pattern recognition receptors (PRR), such as Toll like receptors (TLRs) and nucleotide oligomerization domain (NOD) like receptors (NLRs). TLRs are a family of transmembrane proteins located either at the cell surface or contained within endosomes. Together, the TLR family recognises a wide array of pathogen associated molecular patterns (PAMPs) (Takeda et al., 2003). One example of a TLR that regulates autophagy is TLR2. Infection of murine macrophages with $L$. monocytogenes enhances autophagy, however, this is not evident following infection of TIr2-deficient murine macrophages (Anand et al., 2011). LC3 co-localization with phagocytosed zymosan also requires TLR2 signalling, where LC3 does not co-localize with zymosan in T/r2-deficient macrophages (Sanjuan et al., 2007). LPS treatment of the RAW264.7 macrophage cell line increases GFP-LC3 puncta when visualised by fluorescence microscopy, suggesting autophagy induction (Xu et al., 2007). In this case, initiation of autophagy is dependent on LPS recognition by TLR4, as determined by TIr4 siRNA knockdown or transfection with a mutant dominant-negative TLR4 (Xu et al., 2007). Other examples also demonstrate autophagy induction upon TLR agonist recognition. RAW264.7 macrophages undergo autophagy upon TLR3, 4 and 7 triggering with cognate ligands, as detected by the presence of GFP-LC3 puncta (Delgado et al., 2008). In addition, LC3-II conversion increases upon TLR7 signalling (Delgado et al., 2008). Therefore several TLRs modulate autophagy regulation upon danger signalling.

NLRs recognise bacterial components such as flagellin and peptidoglycan in the cytosol of cells (Benko et al., 2008). NLR triggering can enhance autophagy. NOD2 recognition of muramyldipeptide (a component of peptidoglycan) in human monocyte-derived DC enhances autophagy, as determined by fluorescence microscopy and LC3-I to LC3-II conversion (Cooney et al., 2010). In addition, triggering NOD1 in HeLa cells, and NOD2 in bone marrow-derived macrophages, enhances GFP-LC3 puncta, an observation suggestive of autophagy induction (Travassos et al., 2010). The authors also demonstrate that NOD1 and NOD2 co-immunoprecipitate with Atg16L1, and that NOD-Atg16L1 complexes can be found at the cell surface. The recruitment of NOD-Atg16L1 to the cell surface localises to sites of Shigella flexnari entry into 
into cells, suggesting autophagy may be initiated at sites of pathogen uptake (Travassos et al., 2010). Finally, infection of macrophages with $L$. monocytogenes, upregulates autophagy as evidenced by LC3-II conversion and increases in GFP-LC3 puncta, but this effect is not apparent in Nod2-deficient L. monocytogenes infected macrophages (Anand et al., 2011). Interestingly, some NLR act to inhibit autophagy. The NLR family protein Ipaf, along with Caspase-1 (a downstream target of Ipaf) (Poyet et al., 2001) negatively regulates autophagy induction in bone marrow-derived macrophages upon S. flexnari infection (Suzuki et al., 2007). Macrophages from ipaf- or caspase-1-deficient mice infected with $S$. flexnari exhibit greater levels of autophagy, as measured by LC3-I to LC3-II conversion. Autophagy shutdown with 3-MA in ipaf or caspase-1-deficient macrophages accelerates cell death (Suzuki et al., 2007). The authors propose that in this scenario, NLRs inhibit the pro-survival effects of autophagy in S. flexnari-infected cells to promote cell death and thereby enhance inflammation at the site of infection (Suzuki et al., 2007). Therefore, the data to date suggests that signalling through the NOD receptors regulates autophagy, but that this can occur in both stimulatory and inhibitory contexts with both outcomes acting to promote pathogen clearance.

Cytokines are signalling molecules that can be generated by both immune and non-immune cells for the modulation of immune responses. Like, PPR, cytokines also regulate autophagy. One example of a cytokine that regulates autophagy is IFNy, a pro-inflammatory cytokine. IFNy treatment of RAW264.7 macrophages infected with MTb increases autophagy (Gutierrez et al., 2004; Harris et al., 2007). IFNy treatment increases GFP-LC3 puncta (Gutierrez et al., 2004; Harris et al., 2007) and co-localization of GFP-LC3 with MTb (Gutierrez et al., 2004). MTb survival is reduced following IFNy treatment (Harris et al., 2007), an effect that can be reversed if autophagy is inhibited (Gutierrez et al., 2004). Therefore, autophagy and consequently mycobacterial clearance is enhanced by IFNy. Notably, a stimulatory role for IFNy in autophagy regulation is not always the case. Treatment of HSV-1 infected macrophages with interleukin $1 \beta$ (IL-1 $\beta$ ), but not IFNy, increases autophagy and improves gB-specific $\mathrm{CD}^{+} \mathrm{T}$ cell responses (English et al., 2009). Some cytokines can act as inhibitors of autophagy. Culturing MTb infected RAW264.7 macrophages with interleukin-4 (IL-4) or interleukin 13 (IL-13) inhibits IFNy-mediated autophagy induction, where GFP-LC3 puncta fail to be generated (Harris et al., 2007). IL-4 or IL-13 treatment of infected macrophages also prevents MTb killing (Harris et al., 2007). Therefore, the autophagy pathway is subject to both positive and negative regulation by various stimuli in APC.

\section{CONCLUSIONS}

Autophagy is a major cellular pathway that ensures intracel- lular protein and organelle homeostasis. In undertaking this role, the autophagy machinery intersects with pathways of $\mathrm{MHCl}$ and $\mathrm{MHCll}$ antigen presentation that are required to initiate $T$ cell immunity to pathogen and the selection of $T$ cells in the thymus. Autophagy is therefore a major pathway of host defence and facilitates immune protection from infectious disease. For $\mathrm{MHCll}$ antigen presentation, this intersection largely results from the trafficking of autophagosomes to compartments where $\mathrm{MHCll}$ molecules are loaded with antigen. The contribution of autophagy to MHCll antigen presentation is well established with many examples of antigens being presented by $\mathrm{MHCll}$ in an autophagy-dependent manner. How, or indeed whether, there is a selection mechanism for determining antigen access to the autophagy-dependent route of $\mathrm{MHCll}$ antigen presentation is unknown. For $\mathrm{MHCl}$ antigen presentation, a major contribution for autophagy is less clear, although there are some examples of this occurring for specific viral proteins. Whether this is a relevant pathway of $\mathrm{MHCl}$ presentation for other non-viral antigens or during steady state $\mathrm{MHCl}$ antigen presentation is of interest. How autophagy participates in the presentation of some antigens by $\mathrm{MHCl}$, but not others, remains to be further elucidated. In addition, the putative role of autophagy in $\mathrm{MHCl}$ cross-presentation is intriguing but also requires further investigation. Finally, while it is known that autophagy can be regulated by inflammatory stimuli in APC, the exact signalling mechanisms involved in either suppressing or enhancing autophagy need to be examined in detail. In summary, autophagy is a pathway that clearly intersects with, and contributes to, presentation of antigen via $\mathrm{MHCl}$ and $\mathrm{MHCll}$. As such, pharmacological manipulation of autophagy is a useful strategy to manipulate the presentation of antigen and the induction of robust immunity to fight infection.

\section{ACKNOWLEDGEMENTS}

J.D.M is supported by a National Health and Medical Research Council of Australia Career Development Award.

\section{ABBREVIATIONS}

3-MA, 3-Methyladenine; APC, antigen presenting cell; Atg, autophagy related gene; $\mathrm{CDC}$, conventional dendritic cell; CLIP, class II-associated invariant chain peptide; CMA, chaperone-mediated autophagy; cTEC, cortical thymic epithelial cells; CTL, cytotoxic $T$ lymphocyte; DC, dendritic cell; EBNA-1, Epstein-Barr nuclear antigen 1; EBV, Epstein-Barr virus; ER, endoplasmic reticulum; gB, glycoprotein $B$; GFP, green fluorescent protein; $\mathrm{HA}$, hemagglutinin; HCMV, human cytomegalovirus; HLA, human leukocyte antigen; Hsc70, heat shock cognate protein of $70 \mathrm{kDa}$; HSV, herpes simplex virus; IFNy, interferon gamma; IL, interleukin; LAMP, lysosomal-associated membrane protein ; LC3, microtubule-associated protein light chain 3; MEF, mouse embryonic fibroblast; $\mathrm{MHCl}$, major histocompatibility complex class I; MHCII, major histocompatibility complex class II; 
MIIC, major histocompatibility complex class II loading compartments; MP1, matrix protein 1; MTb, Mycobacterium tuberculosis; mTEC, medullary thymic epithelial cells; mTORC, mammalian target of rapamycin complex; NLR, nucleotide oligomerization domain-like receptor; NOD, nucleotide oligomerization domain; OT-I, CD8 ${ }^{+}$OVAspecific T cells; OT-II, CD4 ${ }^{+}$OVA-specific T cells; OVA, ovalbumin; PAMP, pattern associated molecular pattern; PAS, pre-autophagosomal structure; PE, phosphatidylethanolamine; PI3K, phosphatidylinositol 3-kinase; PRR, pattern recognition receptor; $S 1$, site 1; shRNA, short hairpin RNA; siRNA, small interfering RNA; TAP, transporter associated with antigen processing; TEC, thymic epithelial cells; TLR, Toll like receptor; ULK, unc-51 like kinase; Vps, vacuolar protein sorting

\section{REFERENCES}

Anand, P.K., Tait, S.W., Lamkanfi, M., Amer, A.O., Nunez, G., Pages, G., Pouyssegur, J., McGargill, M.A., Green, D.R., and Kanneganti, T.D. (2011). TLR2 and RIP2 pathways mediate autophagy of Listeria monocytogenes via extracellular signal-regulated kinase (ERK) activation. J Biol Chem 286, 42981-42991.

Baba, M., Takeshige, K., Baba, N., and Ohsumi, Y. (1994). Ultrastructural analysis of the autophagic process in yeast: detection of autophagosomes and their characterization. J Cell Biol 124, 903-913.

Benko, S., Philpott, D.J., and Girardin, S.E. (2008). The microbial and danger signals that activate Nod-like receptors. Cytokine 43, 368-373.

Bevan, M.J. (1976). Cross-priming for a secondary cytotoxic response to minor $\mathrm{H}$ antigens with $\mathrm{H}-2$ congenic cells which do not cross-react in the cytotoxic assay. J Exp Med 143, 1283-1288.

Blommaart, E.F., Krause, U., Schellens, J.P., Vreeling-Sindelarova, H., and Meijer, A.J. (1997). The phosphatidylinositol 3-kinase inhibitors wortmannin and LY294002 inhibit autophagy in isolated rat hepatocytes. Eur J Biochem 243, 240-246.

Burman, C., and Ktistakis, N.T. (2010). Regulation of autophagy by phosphatidylinositol 3-phosphate. FEBS Lett 584, 1302-1312.

Comber, J.D., Robinson, T.M., Siciliano, N.A., Snook, A.E., and Eisenlohr, L.C. (2011). Functional macroautophagy induction by influenza A virus without a contribution to major histocompatibility complex class II-restricted presentation. J Virol 85, 6453-6463.

Cooney, R., Baker, J., Brain, O., Danis, B., Pichulik, T., Allan, P., Ferguson, D.J., Campbell, B.J., Jewell, D., and Simmons, A. (2010). NOD2 stimulation induces autophagy in dendritic cells influencing bacterial handling and antigen presentation. Nat Med $16,90-97$.

De Luca, A., lannitti, R.G., Bozza, S., Beau, R., Casagrande, A., D'Angelo, C., Moretti, S., Cunha, C., Giovannini, G., Massi-Benedetti, C., et al. (2012). CD4(+) T cell vaccination overcomes defective cross-presentation of fungal antigens in a mouse model of chronic granulomatous disease. J Clin Invest 122, 1816-1831.

Delgado, M.A., Elmaoued, R.A., Davis, A.S., Kyei, G., and Deretic, V. (2008). Toll-like receptors control autophagy. EMBO J 27, 1110-1121.

Dengjel, J., Schoor, O., Fischer, R., Reich, M., Kraus, M., Muller, M.,
Kreymborg, K., Altenberend, F., Brandenburg, J., Kalbacher, H., et al. (2005). Autophagy promotes MHC class II presentation of peptides from intracellular source proteins. Proc Natl Acad Sci U S A 102, 7922-7927.

Dorfel, D., Appel, S., Grunebach, F., Weck, M.M., Muller, M.R., Heine, A., and Brossart, P. (2005). Processing and presentation of HLA class I and II epitopes by dendritic cells after transfection with in vitro-transcribed MUC1 RNA. Blood 105, 3199-3205.

English, L., Chemali, M., Duron, J., Rondeau, C., Laplante, A., Gingras, D., Alexander, D., Leib, D., Norbury, C., Lippe, R., et al. (2009). Autophagy enhances the presentation of endogenous viral antigens on MHC class I molecules during HSV-1 infection. Nat Immunol 10, 480-487.

Gutierrez, M.G., Master, S.S., Singh, S.B., Taylor, G.A., Colombo, M.I., and Deretic, V. (2004). Autophagy is a defense mechanism inhibiting BCG and Mycobacterium tuberculosis survival in infected macrophages. Cell 119, 753-766.

Hara, T., Takamura, A., Kishi, C., lemura, S., Natsume, T., Guan, J.L., and Mizushima, N. (2008). FIP200, a ULK-interacting protein, is required for autophagosome formation in mammalian cells. $\mathrm{J}$ Cell Biol 181, 497-510.

Harris, J., De Haro, S.A., Master, S.S., Keane, J., Roberts, E.A., Delgado, M., and Deretic, V. (2007). T helper 2 cytokines inhibit autophagic control of intracellular Mycobacterium tuberculosis. Immunity $27,505-517$.

Jagannath, C., Lindsey, D.R., Dhandayuthapani, S., Xu, Y., Hunter, R.L., Jr., and Eissa, N.T. (2009). Autophagy enhances the efficacy of BCG vaccine by increasing peptide presentation in mouse dendritic cells. Nat Med 15, 267-276.

Jia, W., and He, Y.W. (2011). Temporal regulation of intracellular organelle homeostasis in T lymphocytes by autophagy. J Immunol 186, 5313-5322.

Joffre, O.P., Segura, E., Savina, A., and Amigorena, S. (2012). Cross-presentation by dendritic cells. Nat Rev Immunol 12, 557-569.

Johnstone, C., Ramos, M., Garcia-Barreno, B., Lopez, D., Melero, J.A., and Del Val, M. (2012). Exogenous, TAP-independent lysosomal presentation of a respiratory syncytial virus CTL epitope. Immunol Cell Biol. (In Press).

Jung, C.H., Jun, C.B., Ro, S.H., Kim, Y.M., Otto, N.M., Cao, J., Kundu, M., and Kim, D.H. (2009). ULK-Atg13-FIP200 complexes mediate mTOR signaling to the autophagy machinery. Mol Biol Cell 20, 1992-2003.

Jung, C.H., Ro, S.H., Cao, J., Otto, N.M., and Kim, D.H. (2010). mTOR regulation of autophagy. FEBS Lett 584, 1287-1295.

Kabeya, Y., Mizushima, N., Ueno, T., Yamamoto, A., Kirisako, T., Noda, T., Kominami, E., Ohsumi, Y., and Yoshimori, T. (2000). LC3, a mammalian homologue of yeast Apg8p, is localized in autophagosome membranes after processing. Embo $\mathrm{J} 19$, 5720-5728.

Kasai, M., Tanida, I., Ueno, T., Kominami, E., Seki, S., Ikeda, T., and Mizuochi, T. (2009). Autophagic compartments gain access to the MHC class II compartments in thymic epithelium. J Immunol 183, 7278-7285.

Klionsky, D.J., Cregg, J.M., Dunn, W.A., Jr., Emr, S.D., Sakai, Y., Sandoval, I.V., Sibirny, A., Subramani, S., Thumm, M., Veenhuis, M., et al. (2003). A unified nomenclature for yeast 
autophagy-related genes. Dev Cell 5, 539-545.

Klionsky, D.J., and Ohsumi, Y. (1999). Vacuolar import of proteins and organelles from the cytoplasm. Annu Rev Cell Dev Biol 15, 1-32.

Komatsu, M., Waguri, S., Ueno, T., Iwata, J., Murata, S., Tanida, I., Ezaki, J., Mizushima, N., Ohsumi, Y., Uchiyama, Y., et al. (2005). Impairment of starvation-induced and constitutive autophagy in Atg7-deficient mice. J Cell Biol 169, 425-434.

Kuma, A., Hatano, M., Matsui, M., Yamamoto, A., Nakaya, H., Yoshimori, T., Ohsumi, Y., Tokuhisa, T., and Mizushima, N. (2004). The role of autophagy during the early neonatal starvation period. Nature 432, 1032-1036.

Lee, H.K., Mattei, L.M., Steinberg, B.E., Alberts, P., Lee, Y.H., Chervonsky, A., Mizushima, N., Grinstein, S., and Iwasaki, A. (2010). In vivo requirement for Atg5 in antigen presentation by dendritic cells. Immunity 32, 227-239.

Levine, B., and Kroemer, G. (2008). Autophagy in the pathogenesis of disease. Cell 132, 27-42.

Li, B., Lei, Z., Lichty, B.D., Li, D., Zhang, G.M., Feng, Z.H., Wan, Y., and Huang, B. (2010). Autophagy facilitates major histocompatibility complex class I expression induced by IFN-gamma in B16 melanoma cells. Cancer Immunol Immunother 59, 313-321.

Li, H., Li, Y., Jiao, J., and Hu, H.M. (2011a). Alpha-alumina nanoparticles induce efficient autophagy-dependent cross-presentation and potent antitumour response. Nature nanotechnology 6, 645-650.

Li, W., Yang, Q., and Mao, Z. (2011b). Chaperone-mediated autophagy: machinery, regulation and biological consequences. Cell Mol Life Sci 68, 749-763.

Li, Y., Wang, L.X., Yang, G., Hao, F., Urba, W.J., and Hu, H.M. (2008). Efficient cross-presentation depends on autophagy in tumor cells. Cancer research 68, 6889-6895.

McLeod, I.X., Zhou, X., Li, Q.J., Wang, F., and He, Y.W. (2011). The class III kinase Vps34 promotes $T$ lymphocyte survival through regulating IL-7Ralpha surface expression. J Immunol 187, 5051-5061.

Mintern, J.D., and Villadangos, J.A. (2012). Autophagy and mechanisms of effective immunity. Front Immunol 3, 60.

Mizushima, N., Noda, T., Yoshimori, T., Tanaka, Y., Ishii, T., George, M.D., Klionsky, D.J., Ohsumi, M., and Ohsumi, Y. (1998). A protein conjugation system essential for autophagy. Nature 395, 395-398.

Mizushima, N., Yamamoto, A., Matsui, M., Yoshimori, T., and Ohsumi, Y. (2004). In vivo analysis of autophagy in response to nutrient starvation using transgenic mice expressing a fluorescent autophagosome marker. Mol Biol Cell 15, 1101-1111.

Nedjic, J., Aichinger, M., Emmerich, J., Mizushima, N., and Klein, L. (2008). Autophagy in thymic epithelium shapes the T-cell repertoire and is essential for tolerance. Nature 455, 396-400.

Nimmerjahn, F., Milosevic, S., Behrends, U., Jaffee, E.M., Pardoll, D.M., Bornkamm, G.W., and Mautner, J. (2003). Major histocompatibility complex class II-restricted presentation of a cytosolic antigen by autophagy. Eur J Immunol 33, 1250-1259.

Ohsumi, Y. (2001). Molecular dissection of autophagy: two ubiquitin-like systems. Nat Rev Mol Cell Biol 2, 211-216.

Orenstein, S.J., and Cuervo, A.M. (2010). Chaperone-mediated autophagy: molecular mechanisms and physiological relevance. Semin Cell Dev Biol 21, 719-726.

Orsi, A., Razi, M., Dooley, H.C., Robinson, D., Weston, A.E., Collinson, L.M., and Tooze, S.A. (2012). Dynamic and transient interactions of Atg9 with autophagosomes, but not membrane integration, are required for autophagy. Mol Biol Cell 23, 1860-1873.

Paludan, C., Schmid, D., Landthaler, M., Vockerodt, M., Kube, D., Tuschl, T., and Munz, C. (2005). Endogenous MHC class II processing of a viral nuclear antigen after autophagy. Science 307 593-596.

Poyet, J.L., Srinivasula, S.M., Tnani, M., Razmara, M. Fernandes-Alnemri, T., and Alnemri, E.S. (2001). Identification of Ipaf, a human caspase-1-activating protein related to Apaf-1. J Biol Chem 276, 28309-28313.

Qu, X., Zou, Z., Sun, Q., Luby-Phelps, K., Cheng, P., Hogan, R.N., Gilpin, C., and Levine, B. (2007). Autophagy gene-dependent clearance of apoptotic cells during embryonic development. Cell 128, 931-946.

Riedel, A., Nimmerjahn, F., Burdach, S., Behrends, U., Bornkamm, G.W., and Mautner, J. (2008). Endogenous presentation of a nuclear antigen on MHC class II by autophagy in the absence of CRM1-mediated nuclear export. Eur J Immunol 38, 2090-2095.

Saitoh, T., Fujita, N., Hayashi, T., Takahara, K., Satoh, T., Lee, H., Matsunaga, K., Kageyama, S., Omori, H., Noda, T., et al. (2009). Atg9a controls dsDNA-driven dynamic translocation of STING and the innate immune response. Proc Natl Acad Sci U S A 106, 20842-20846.

Saitoh, T., Fujita, N., Jang, M.H., Uematsu, S., Yang, B.G., Satoh, T., Omori, H., Noda, T., Yamamoto, N., Komatsu, M., et al. (2008). Loss of the autophagy protein Atg16L1 enhances endotoxin-induced IL-1beta production. Nature 456, 264-268.

Sanjuan, M.A., Dillon, C.P., Tait, S.W., Moshiach, S., Dorsey, F., Connell, S., Komatsu, M., Tanaka, K., Cleveland, J.L., Withoff, S., et al. (2007). Toll-like receptor signalling in macrophages links the autophagy pathway to phagocytosis. Nature 450 , 1253-1257.

Schmid, D., Pypaert, M., and Munz, C. (2007). Antigen-loading compartments for major histocompatibility complex class II molecules continuously receive input from autophagosomes. Immunity 26, 79-92.

Seglen, P.O., and Gordon, P.B. (1982). 3-Methyladenine: specific inhibitor of autophagic/lysosomal protein degradation in isolated rat hepatocytes. Proc Natl Acad Sci U S A 79, 1889-1892.

Segura, E., and Villadangos, J.A. (2011). A modular and combinatorial view of the antigen cross-presentation pathway in dendritic cells. Traffic 12, 1677-1685.

Shintani, T., Mizushima, N., Ogawa, Y., Matsuura, A., Noda, T., and Ohsumi, Y. (1999). Apg10p, a novel protein-conjugating enzyme essential for autophagy in yeast. Embo J 18, 5234-5241.

Sou, Y.S., Waguri, S., Iwata, J., Ueno, T., Fujimura, T., Hara, T., Sawada, N., Yamada, A., Mizushima, N., Uchiyama, Y., et al. (2008). The Atg8 conjugation system is indispensable for proper development of autophagic isolation membranes in mice. Mol Biol Cell 19, 4762-4775.

Sukseree, S., Mildner, M., Rossiter, H., Pammer, J., Zhang, C.F., Watanapokasin, R., Tschachler, E., and Eckhart, L. (2012). 
Autophagy in the thymic epithelium is dispensable for the development of self-tolerance in a novel mouse model. PLoS One 7, e38933.

Suzuki, K., and Ohsumi, Y. (2007). Molecular machinery of autophagosome formation in yeast, Saccharomyces cerevisiae. FEBS Lett 581, 2156-2161.

Suzuki, T., Franchi, L., Toma, C., Ashida, H., Ogawa, M., Yoshikawa, Y., Mimuro, H., Inohara, N., Sasakawa, C., and Nunez, G. (2007). Differential regulation of caspase-1 activation, pyroptosis, and autophagy via Ipaf and ASC in Shigella-infected macrophages. PLoS Pathog 3, e111.

Takeda, K., Kaisho, T., and Akira, S. (2003). Toll-like receptors. Annu Rev Immunol 21, 335-376.

Tanida, I., Ueno, T., and Kominami, E. (2004). LC3 conjugation system in mammalian autophagy. Int J Biochem Cell Biol 36, 2503-2518.

Tey, S.K., and Khanna, R. (2012). Autophagy mediates transporter associated with antigen processing-independent presentation of viral epitopes through MHC class I pathway. Blood 120, 994-1004.

Travassos, L.H., Carneiro, L.A., Ramjeet, M., Hussey, S., Kim, Y.G., Magalhaes, J.G., Yuan, L., Soares, F., Chea, E., Le Bourhis, L., et al. (2010). Nod1 and Nod2 direct autophagy by recruiting
ATG16L1 to the plasma membrane at the site of bacterial entry. Nat Immunol 11, 55-62.

Uhl, M., Kepp, O., Jusforgues-Saklani, H., Vicencio, J.M., Kroemer, G., and Albert, M.L. (2009). Autophagy within the antigen donor cell facilitates efficient antigen cross-priming of virus-specific CD8+ T cells. Cell death and differentiation 16, 991-1005.

Weidberg, H., Shvets, E., and Elazar, Z. (2011). Biogenesis and cargo selectivity of autophagosomes. Annu Rev Biochem 80, 125-156.

Willinger, T., and Flavell, R.A. (2012). Canonical autophagy dependent on the class III phosphoinositide-3 kinase Vps34 is required for naive T-cell homeostasis. Proc Natl Acad Sci U S A 109, 8670-8675.

Xu, Y., Jagannath, C., Liu, X.D., Sharafkhaneh, A., Kolodziejska, K.E., and Eissa, N.T. (2007). Toll-like receptor 4 is a sensor for autophagy associated with innate immunity. Immunity 27 , 135-144.

Yang, Z., and Klionsky, D.J. (2010). Eaten alive: a history of macroautophagy. Nat Cell Biol 12, 814-822.

Zhou, D., Li, P., Lin, Y., Lott, J.M., Hislop, A.D., Canaday, D.H., Brutkiewicz, R.R., and Blum, J.S. (2005). Lamp-2a facilitates MHC class II presentation of cytoplasmic antigens. Immunity 22, 571-581. 